\title{
IL-4 inhibits regulatory $T$ cells differentiation by HDAC9-mediated epigenetic regulation
}

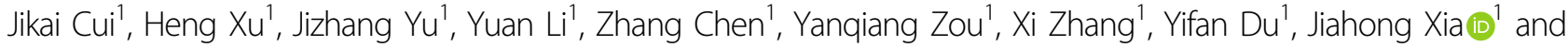 \\ Jie Wu ${ }^{1}$
}

\begin{abstract}
Regulatory $T$ cells play a crucial role in orchestrating immune response and maintaining immune tolerance, and the expression of the Foxp3 gene is indispensable to the differentiation of regulatory T cells. IL-4 shows strong inhibitory effects on Foxp3 expression and regulatory T cells differentiation, but the detailed mechanisms are still unclear. Here, we revealed that epigenetic modulations are key to this process. Specifically, the inhibition was found to be STAT6 dependent, and HDAC9 was involved with the process of histone deacetylation at the Foxp3 locus, subsequently decreasing chromatin accessibility and Foxp3 gene transcription. Pan-histone deacetylation inhibitors, especially sodium butyrate, notably abolished the inhibitory effects of IL-4 and ameliorated allergic airway inflammation in mouse models. Our research provides important mechanistic insights into how IL-4 inhibits regulatory T cells differentiation and suggests the therapeutic potential of the sodium butyrate in allergic airway disease.
\end{abstract}

\section{Introduction}

Regulatory $\mathrm{T}$ cells (Tregs) play a crucial role in orchestrating immune response and maintaining immune tolerance ${ }^{1}$. Both natural Tregs (nTregs) and inducible Tregs (iTregs) express the master transcriptional regulator Foxp3(ref. ${ }^{2,3}$ ). Foxp3 deficiency causes fatal aggressive autoimmunity in mice ${ }^{4}$, and its mutation leads to immunodysregulation, polyendocrinopathy, enteropathy, X-linked syndrome (IPEX) in humans ${ }^{5}$. Treg differentiation in vitro requires TGF- $\beta$ and IL-2(ref. ${ }^{6,7}$ ), which effectively induces Foxp3 expression. However, Tregs are quite unstable and easily to lose Foxp3 expression $^{8}$; for example, the inflammatory cytokine IL-6 prevents Tregs differentiation but boosts Th17 generation ${ }^{9}$. It has also been found that IL-4 suppresses Tregs differentiation ${ }^{10}$. Rivas et al. found that enhancing IL-4RSTAT6 signaling decreased the formation of allergen-

\footnotetext{
Correspondence: Jiahong Xia (jiahong.xia@hust.edu.cn) or Jie Wu (wujie426@hust.edu.cn)

${ }^{1}$ Department of Cardiovascular Surgery, Union Hospital, Tongji Medical College, Huazhong University of Science and Technology, Wuhan 430022, China

These authors contributed equally: Jikai Cui, Heng Xu, Jizhang Yu. Edited by H.-U. Simon
}

specific Treg in food allergy model ${ }^{11}$. IL- 4 secreted by Th2 is enriched in allergic asthma ${ }^{12}$ and are potent at promoting allergic inflammation ${ }^{13}$. However, the underlying mechanisms of IL-4 repressing Treg differentiation have not been clarified.

Recently, epigenetic factors have been shown to be involved in the regulation of the plasticity and specificity of $\mathrm{T}$ cell subsets ${ }^{14}$. Gene expression is epigenetically regulated through chromatin modifications such as histone methylation, acetylation, and other covalent ways ${ }^{15}$. Generally, histone acetyltransferases increase chromatin accessibility and promote gene transcription, whereas histone deacetylases (HDACs) typically repress gene transcription, although exceptions do exist ${ }^{16}$. HDAC inhibitor $(\mathrm{HDACi})$ restrains the process of histone deacetylation and promotes gene transcription ${ }^{17}$. Independent of the known anticancer effects of HDACi, such as proapoptotic activity or cell cycle arrest induction ${ }^{18}$, the anti-inflammatory functions of these agents have recently aroused interest ${ }^{19}$. Previous researches have showed that epigenetic regulation play roles in Treg stability ${ }^{20}$; for example, the HDACi trichostatin A (TSA) prevented the differentiation of human Foxp3 ${ }^{+}$Tregs into IL-17

\section{(c) The Author(s) 2021}

(c) (i) Open Access This article is licensed under a Creative Commons Attribution 4.0 International License, which permits use, sharing, adaptation, distribution and reproduction cc) in any medium or format, as long as you give appropriate credit to the original author(s) and the source, provide a link to the Creative Commons license, and indicate if changes were made. The images or other third party material in this article are included in the article's Creative Commons license, unless indicated otherwise in a credit line to the material. If material is not included in the article's Creative Commons license and your intended use is not permitted by statutory regulation or exceeds the permitted use, you will need to obtain permission directly from the copyright holder. To view a copy of this license, visit http://creativecommons.org/licenses/by/4.0/. 
producing cells ${ }^{21}$. Among the multiple HDACs Treg expresses, HDAC9 was proved to play an important role in regulating Foxp3-dependent suppression ${ }^{22}$. Moreover, HDAC9 was upregulated in colitis and $\mathrm{HDAC}^{-1-}$ mice was resistant to develop colitis ${ }^{23}$. Therefore, it is necessary to clarify whether epigenetic factors are involved in the process of IL-4-induced inhibition of Treg differentiation.

In this study, we found that Treg differentiation inhibited by IL-4 is STAT6 dependent, and HDAC9 was involved in the process of histone deacetylation at the Foxp3 locus, decreasing chromatin accessibility and Foxp3 gene transcription. As a pan-HDACi, sodium butyrate $(\mathrm{NaB})$ efficiently abrogates the effect of IL- 4 and ameliorates allergic airway inflammation in vivo. These results explain the mechanisms underlying IL-4-induced inhibition of Treg differentiation and suggest the therapeutic potential of the pan-HDACi NaB in asthma.

\section{Results}

IL-4 inhibits TGF- $\beta$-mediated Foxp $3^{+}$T cell differentiation

Activated with TGF- $\beta 1$ and IL-2, up to $80-90 \%$ of naive $\mathrm{T}$ cells differentiated into Foxp $3^{+}$Tregs; in contrast, in the presence of IL-4, this percentage declined to $~ 15 \%$ (Fig. 1A). To study the underlying mechanisms, we first examined the transcriptional changes on the second day of induction. The results showed that IL-4 observably decreased the mRNA level of Foxp3, indicating that IL-4 influenced the transcription of Foxp3. Meanwhile, the mRNA levels of Gata3 and Il9 markedly increased, and the Il4 mRNA level slightly changed, but this difference was not significant (Fig. 1B).

\section{IL-4 triggers chromatin remodeling at the Foxp3 locus}

Recent studies have found that numerous genes, including Foxp3, are under the regulation of epigenetic mechanisms $^{22}$. To study whether epigenetic changes play a part in Foxp3 transcription inhibition by IL-4, we used ATAC-seq to evaluate the open chromatin status of the Foxp3 locus. Under Treg-polarizing conditions, the Foxp3 locus, especially the promoter, CNS1 and CNS2 regions, were highly accessible; however, the addition of IL-4 "closed" these regions (Fig. 1C). This chromatin remodeling prevents the transcription machinery from binding with the Foxp3 locus.

\section{IL-4 reduces histone acetylation at the Foxp3 locus}

To further determine which epigenetic modification plays a major role in this process, we used ChIP-qPCR to analyze histone methylation and acetylation at the Foxp3 locus. We focused on several classic modifications including H3Ac, H4Ac, H3K9Ac, H3K27Ac, H3K9Me2, H3K9Me3, H3K27Me2, and H3K27Me3. Compared to standard Treg-polarizing conditions, the addition of IL-4 significantly decreased the studied histone acetylation modifications at the Foxp3 locus, including in the promoter, CNS1 and CNS2 regions (Fig. 1D). In contrast, IL-4 did not obviously alter the studied histone methylation modifications at the Foxp3 locus (Fig. 1E). Hence, chromatin remodeling triggered by IL- 4 is the result of histone deacetylation modifications.

\section{IL-4-induced Foxp3 repression is attenuated by $\mathrm{NaB}$ (pan- HDACi)}

Knowing that histone deacetylation plays a key role in Foxp3 inhibition induced by IL-4, we used HDAC inhibitors to determine whether histone deacetylation is indispensable. The results showed that several pan-HDACi and class II HDACi dramatically rescued the Foxp3 expression inhibition induced by IL-4 (Fig. 2A); in particular, $\mathrm{NaB}$ almost eliminated the effect of IL-4 (No IL-4: 85\%; IL-4: $\sim 15 \%$; IL-4 + NaB: $75 \%$ ), and the effect of $\mathrm{NaB}$ increased as the $\mathrm{NaB}$ concentration increased (Fig. 2B). However, other more specific HDACi tested showed slight effects. Notably, the addition of $\mathrm{NaB}$ did not affect the expression of GATA3 (Fig. 2C), indicating that this process is independent of GATA3. Moreover, ChIP-qPCR showed that $\mathrm{NaB}$ significantly rescued the $\mathrm{H} 3, \mathrm{H} 4, \mathrm{H} 3 \mathrm{~K} 9$, and H3K27 acetylation of the Foxp3 locus, including the promoter, CNS1 and CNS2 regions (Fig. 2D). These data suggest that the pan-HDACi $\mathrm{NaB}$ can effectively rescue the Foxp3 inhibition induced by IL-4.

\section{The HDAC inhibitor NaB ameliorates allergic airway inflammation in vivo}

In allergic airway inflammation, IL-4 is an important inflammatory cytokine, while Tregs negatively regulate the intensity of the inflammatory response ${ }^{24}$. We established an OVA-induced acute allergic lung inflammation mouse model to study the effect of $\mathrm{NaB}$ in vivo (Fig. 3A). Through analysis of histological changes in the lungs, we found that treatment with $\mathrm{NaB}$ observably ameliorated inflammatory cell infiltration and mucin-secreting cell $\left(\mathrm{PAS}^{+}\right)$hyperplasia around the airways, which was in accordance with the results of quantitative assessments (Fig. 3B, D). FCM analysis showed that the percentage of eosinophils among $\mathrm{F} 4 / 80^{+}$lung infiltrated cells declined markedly after $\mathrm{NaB}$ treatment (PBS: $\sim 40 \%$; $\mathrm{NaB}: \sim 10 \%$ ) (Fig. 3C, D $)^{25}$. Moreover, $\mathrm{NaB}$ treatment decreased the infiltrated CD45, $\mathrm{T}$ and $\mathrm{B}$ cells and led to reduced percent of IL-4 secreting T cells and more Treg, along with alleviative activation and proliferation of lung infiltrated $\mathrm{T}$ and $\mathrm{B}$ cells (sFig 1). Similarly, $\mathrm{NaB}$ treatment reduced the numbers of total and specific inflammatory cell populations in the BAL fluid (Fig. 3E), along with reduced Th2 cytokine protein levels in the BAL fluid and reduced serum total IgE level (Fig. 3F). Together, these results demonstrated that $\mathrm{NaB}$ ameliorated allergic airway inflammation in a mouse model of OVA-induced acute allergy. 
A

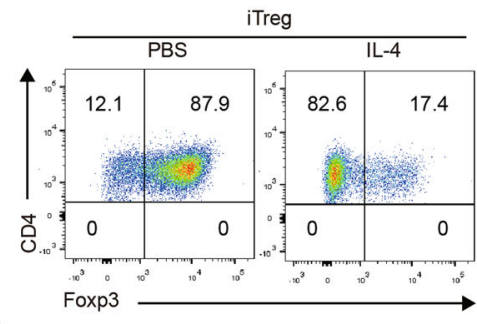

C

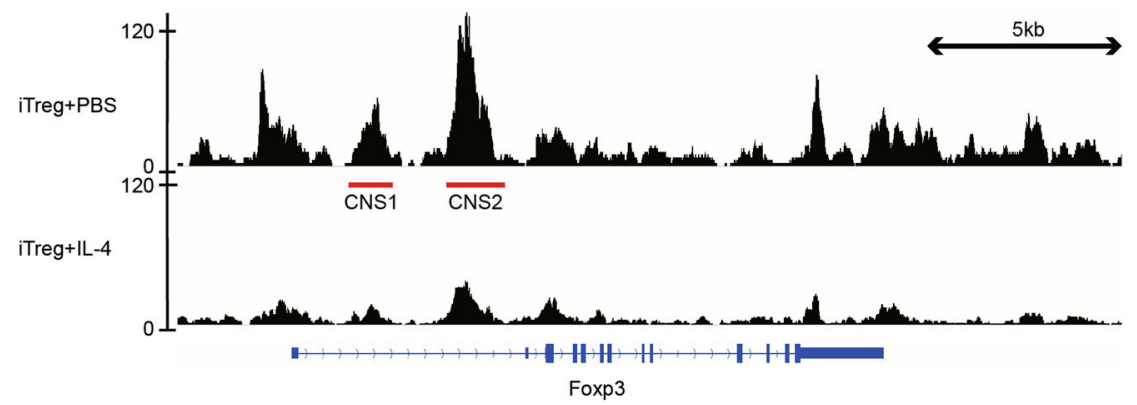

B
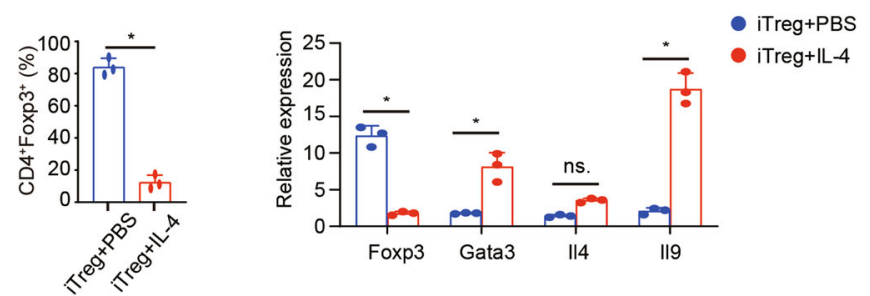

D

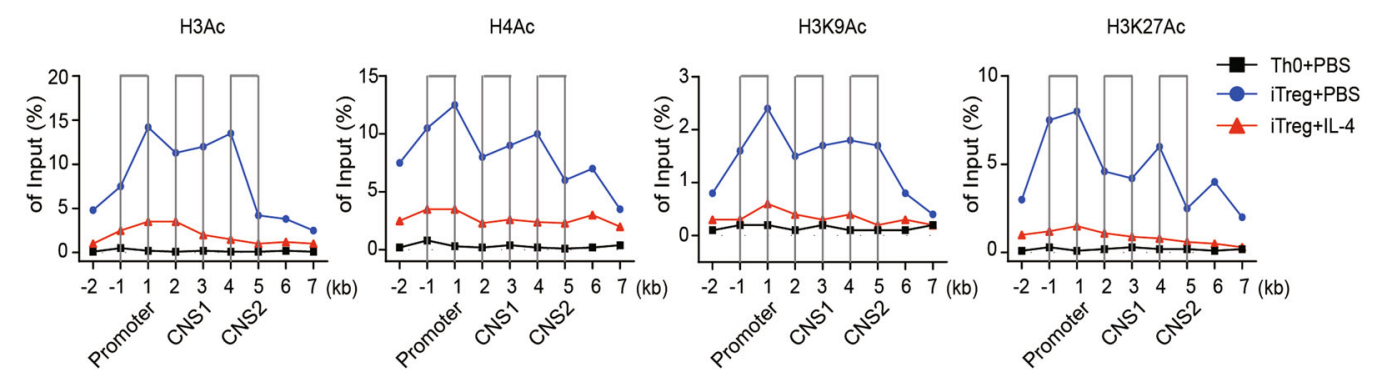

E
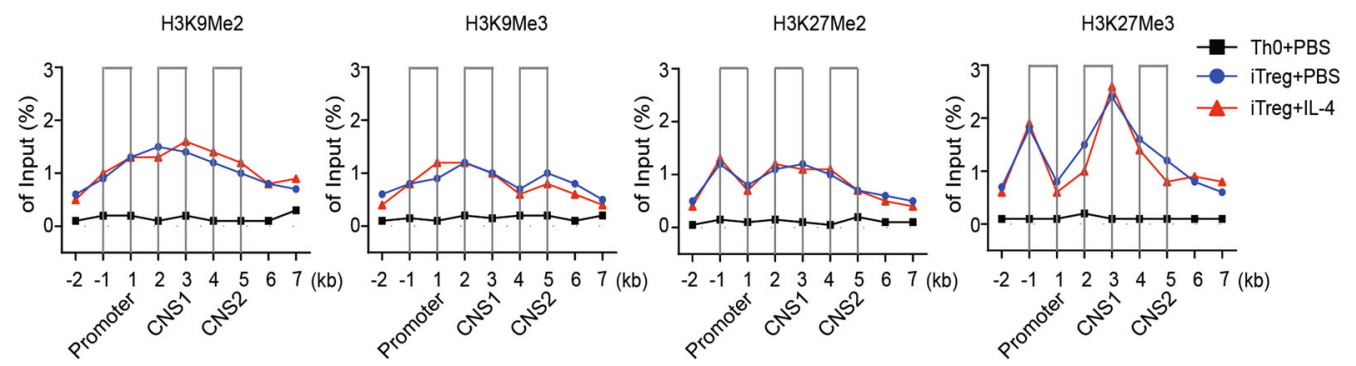

Fig. 1 IL-4 inhibits Foxp3 transcription and histone acetylation of Foxp3 locus. Naive CD4 ${ }^{+} \mathrm{T}$ cells from WT mice were cultured under Tregpolarizing conditions with or without IL-4. A Cells were harvested 3 days later and analyzed by FCM. The percentages of Foxp3 ${ }^{+}$cells are displayed in the quadrants. B On day 2, cells were harvested and analyzed by quantitative RT-PCR. The graphs display the relative mRNA expression of Foxp3, Gata3, II9, and II4. C On day 2, cells were prepared for ATAC-seq. Integrative Genomics Viewer (IGV) screenshots of representative ATAC-seq tracks from each group at the Foxp3 locus are shown. Red bands represent classic regulatory regions. The scales of the two tracks were adjusted to display comparable intensities, and the data are representative of two biologically independent pooled samples. D, E ChIP-qPCR analyses for H3Ac, H4Ac, H3K27Ac, H3K9Ac, H3K9Me2, H3K9Me3, H3K27Me2, and H3K27Me3 alterations at the Foxp3 locus were performed. Data are the mean \pm SD and representative of three independent experiments. ${ }^{*} p<0.05$.

\section{IL-4-induced inhibition of Foxp3 transcription depends on} STAT6

In the process of Th2 cell differentiation, IL-4 activates STAT6 after binding with IL-4 receptor ${ }^{26}$.
To determine whether STAT6 is involved in IL-4induced suppression of Foxp3 transcription, we cultured naive $\mathrm{CD} 4^{+} \mathrm{T}$ cells from Stat $6^{-/-}$mice under Treg induction conditions with or without IL-4. Strikingly, 
A

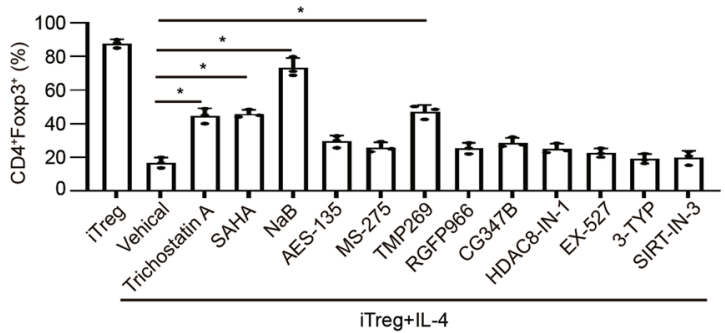

B

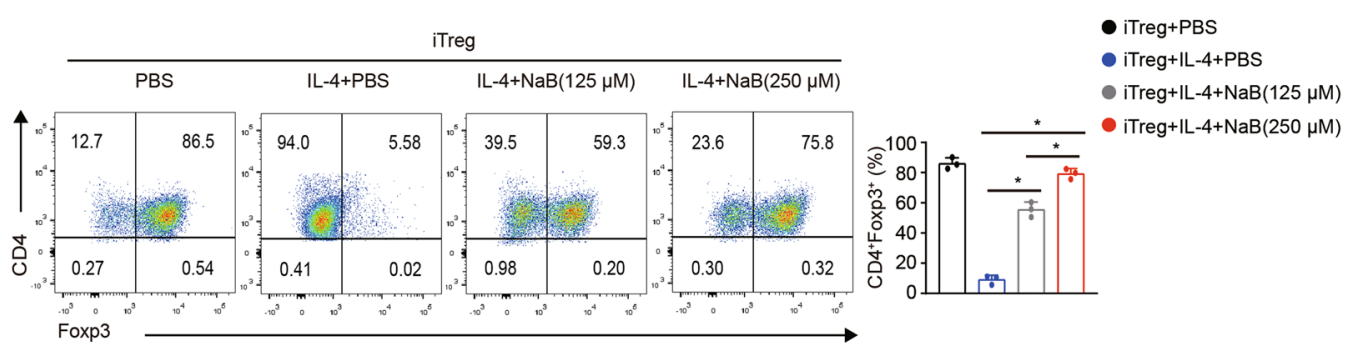

C
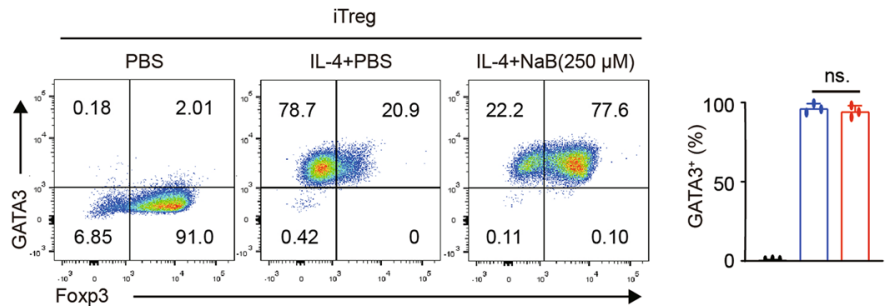

- iTreg+PBS

- iTreg+IL-4+PBS

- iTreg+IL-4+NaB(250 $\mu \mathrm{M})$

D
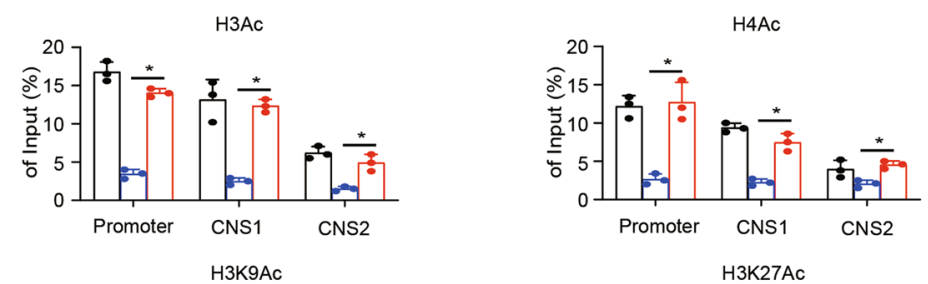

- iTreg+PBS

- iTreg+IL-4+PBS

- ITreg+LL-4+NaB(250 $\mu \mathrm{M})$
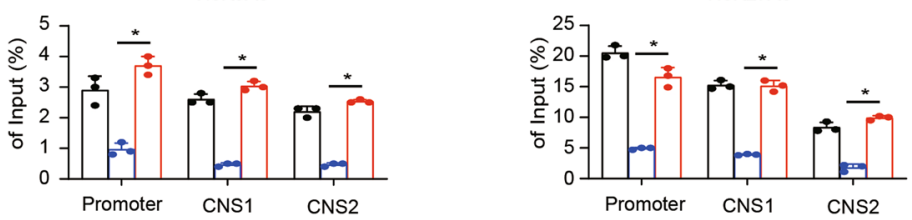

Fig. 2 IL-4-induced Foxp3 repression is attenuated by $\mathrm{NaB}$ (pan-HDAC inhibitor). Naive CD4 ${ }^{+} \mathrm{T}$ cells from WT B6 mice were cultured under Treg-polarizing conditions with or without IL-4 and inhibitors for 2 (D) or 3 (A, B, C) days. A The following inhibitors were added at various

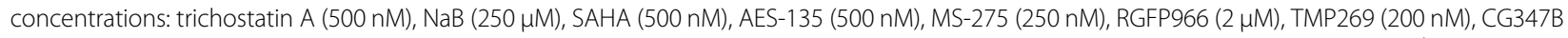
(200 nM), HDAC8-IN-1 (200 nM), EX-527 (100 nM), 3-TYP (20 nM), SIRT-IN-3 $(50 \mu \mathrm{M})$. The histogram displays the percentages of Foxp3 ${ }^{+}$T cells in different groups. B, C The color FACS plots depict Foxp ${ }^{+}$and $\mathrm{GATA}^{+}$cells. The percentages of different cells are displayed in the quadrants. D ChIPqPCR assays for H3Ac, H4Ac, H3K27Ac and H3K9Ac modifications in the Foxp3 promoter, CNS1, and CNS2 regions were performed. Data are the mean $\pm S D$ and representative of three independent experiments. ${ }^{*} p<0.05$.

most of the cells $(85 \%)$ differentiated into Foxp $3^{+}$Tregs, even in the presence of IL-4, and the percentage of IL-9secreting cells did not increase (Fig. 4A). Moreover, ChIP-qPCR showed that the $\mathrm{H} 3$ and $\mathrm{H} 4$ acetylation of the Foxp3 locus, including the promoter, CNS1 and CNS2 regions remained unchanged (Fig. 4B). Therefore, STAT6 plays an indispensable role in the process of IL-4 suppressing Foxp3 transcription. 
A

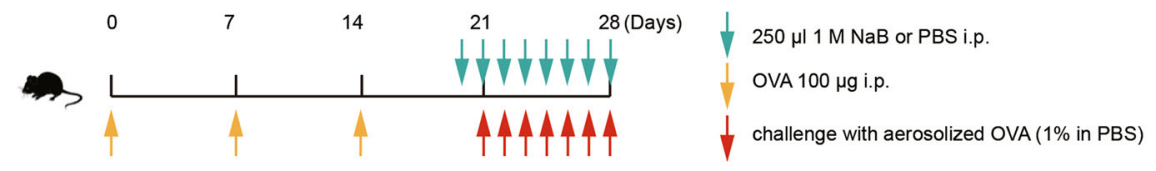

B
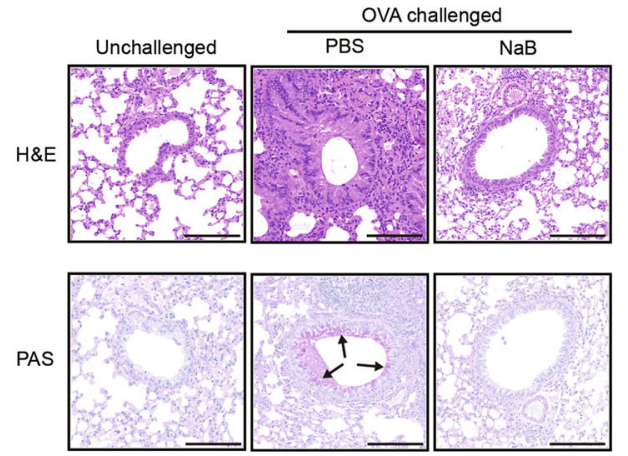

E

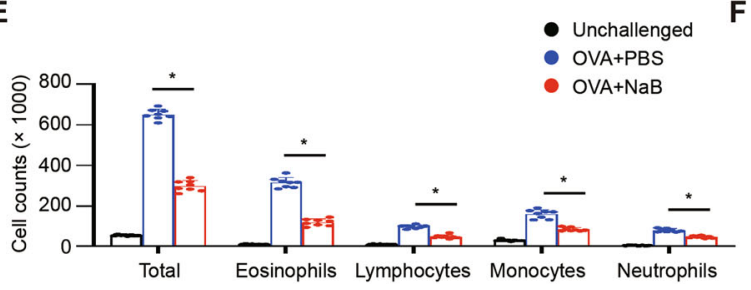

C

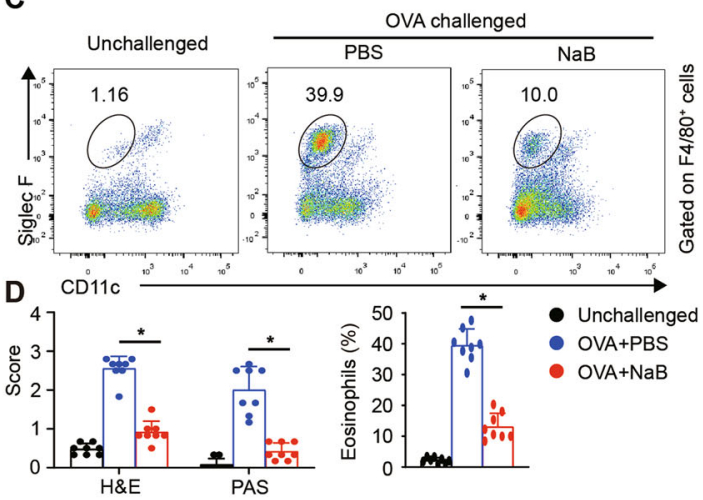

$\mathbf{F}$

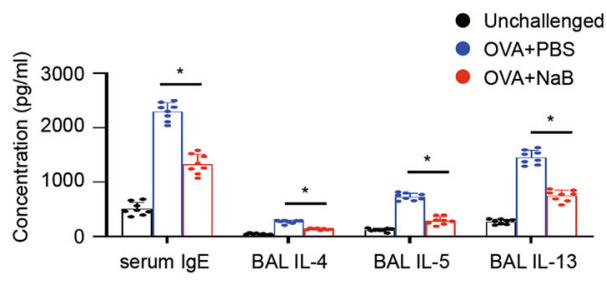

Fig. $3 \mathrm{NaB}$ ameliorates allergic airway inflammation in vivo. A Schematic representation of the OVA-induced acute allergic lung inflammation model. B Representative lung histology sections displaying inflammatory cell infiltration and AAS $^{+}$mucin-secreting cell hyperplasia. The arrow indicates purple-red mucin-producing cells. Bars, $100 \mu \mathrm{m}$. C Gate in color FACS plots identifying infiltrated eosinophils $\left(C D 45^{+} \mathrm{F} 4 / 80^{+}\right.$Siglec

$\mathrm{F}^{+} \mathrm{CD} 11 \mathrm{c}^{-}$) in the lungs. D The graph displays the histological scores determined by H\&E and PAS staining. Five areas were randomly chosen in each slide. The percentages of eosinophils among $\mathrm{F} 4 / 80^{+}$lung infiltrated cells are displayed in the histograms. E Different cell counts in the BAL fluid. $\mathbf{F}$ Total IgE levels in the blood serum and IL-4, IL-5, and IL-13 levels in the BAL fluid, as determined by ELISA. Data are the mean \pm SD and representative of three independent experiments. Each group included eight mice. ${ }^{*} p<0.05$.

HDAC9 is involved in IL-4-STAT6-mediated epigenetic changes at the Foxp3 locus

To specifically identify the HDAC involved in IL-4STAT6-mediated epigenetic suppression, we screened the mRNA changes in all HDAC types and found that the Hdac9 mRNA level obviously increased with the addition of IL-4 (Fig. 4C). Western-blotting assay verified this change at the protein level (Fig. 4D, E). However, this increase was not observed in Stat $6^{-1-} \mathrm{T}$ cells, further indicating that HDAC9 is downstream of the IL-4-STAT6 pathway. We further showed that HDAC9 was enriched at the Foxp3 locus under the Treg plus IL-4 condition, which wasn't been observed in Stat6-deficient cells (Fig. $5 \mathrm{~A})$. Because of the lack of a specific inhibitor targeting HDAC9, we used shRNA to specifically knockdown Hdac9 expression in $\mathrm{T}$ cells, which was verified at the protein level with Western-blotting assay (sFig 4A). The FCM results showed that Hdac9 knockdown strikingly decreased the inhibitory effect of IL-4 on the Treg polarization (Fig. 5B). Moreover, ChIP-qPCR showed that
Hdac9 knockdown significantly rescued the $\mathrm{H} 3$ and $\mathrm{H} 4$ acetylation of the Foxp3 locus, including the promoter, CNS1 and CNS2 regions (Fig. 5C). Taken together, our data suggest that HDAC9 is recruited to the Foxp3 locus under Treg plus IL-4 condition, and STAT6 is crucial in this process.

\section{Hdac9 knockdown alleviates allergic airway inflammation and increases Treg proportion in an OT-II cell adoptive transfer model}

To further study the role of HDAC9 in vivo, we selectively knockdown Hdac9 expression with an Hdac9specific shRNA (or shRNA controls) in OT-II cells and established adoptive transfer models (Fig. 6A). After daily aerosolization with OVA (5\% in PBS) for 7 days, the transferred mice were sacrificed for histological analysis. As shown in Fig. 6B, Hdac9 knockdown in OT-II cells significantly decreased the infiltration of inflammatory cells and proliferation of mucin-secreting cells $\left(\mathrm{PAS}^{+}\right)$ around the airways, which was in accordance with the 
A

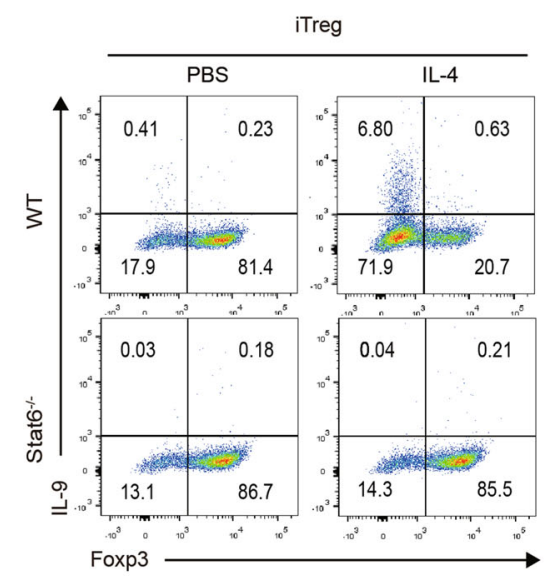

B

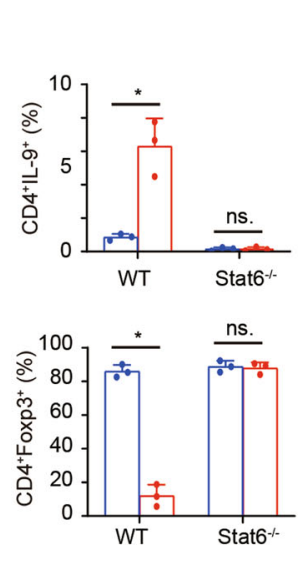

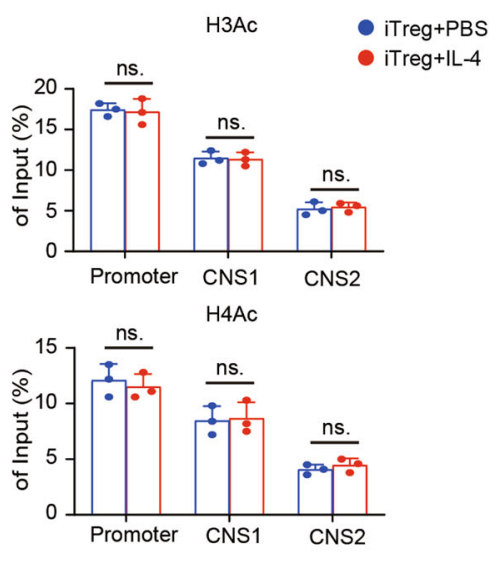

C

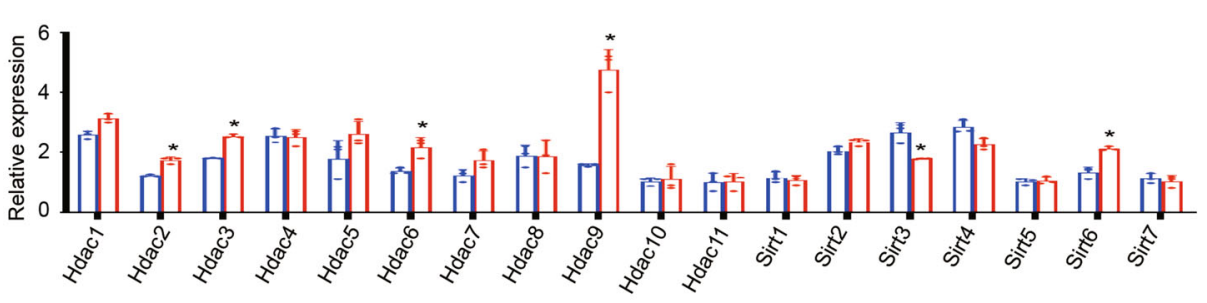

D

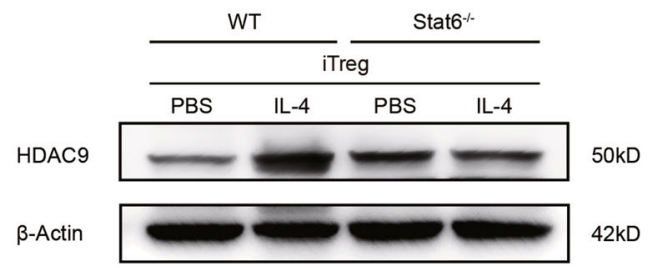

E

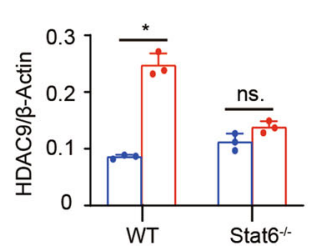

Fig. 4 HDAC9 is involved in IL-4-STAT6-mediated epigenetic changes. A Naive CD4 ${ }^{+} T$ cells were FACS sorted from WT B6 and stat6 $6^{-1-}$ mice and cultured under iTreg-polarizing conditions with or without IL-4. The cells were harvested on day 2 (B, C) or day 3 (A, D). A An FCM assay was used to measure the percentages of Foxp $3^{+}$cells among $\mathrm{CD}^{+}{ }^{+} \mathrm{T}$ cells in different group. B ChIP-qPCR assays for H3Ac and H4Ac modifications in the Foxp3 promoter, CNS1, and CNS2 regions with T cells from stat6 ${ }^{-/}$mice. C The graphs display Hdac1-11 and Sirt1-7 mRNA expression in the cultured cells relative to that in naive $\mathrm{CD}^{+}$T cells. D An immunoblot assay evaluating HDAC9 expression was performed. E The graphs show quantitation of HDAC9 expression in three independent western blot experiments. Data are the mean \pm SD and representative of three independent experiments. ${ }^{*} p<0.05$.

disease scores calculated. Moreover, several Th2 cytokine protein levels in the BAL fluid and serum total IgE level decreased in Hdac9 knockdown group (Fig. 6C). Then we established CD45.1/2 $2^{+}$sh-Ctrl/Hdac9 OT-II cell transfer allergy model (Fig. 6D). Analyzing the lymphocyte infiltrated in lungs (Fig. 6E), we found the percentage of Tregs is higher in Hdac9 knockdown cells, compared with the control cells (Fig. 6F), suggesting that inhibiting HDAC9 contributes to the stability of Tregs in lungs. Collectively, these data showed that knockdown Hdac9 could alleviates allergic airway inflammation, which was correlated with the increased Treg proportion.

\section{Discussion}

IL-4 is known to inhibit naive $\mathrm{CD} 4^{+} \mathrm{T}$ cell differentiation into Foxp $3^{+}$Tregs; however, the specific mechanisms underlying this phenomenon are still unclarified. In this article, we found that epigenetic regulation triggered by IL-4 plays a key role in this process. HDAC9 is involved in chromatin modifications by regulating histone acetylation at the Foxp3 locus, and STAT6 plays a crucial role in this process. The pan-HDAC inhibitor $\mathrm{NaB}$ efficiently abrogates the inhibitory effect of IL-4 and ameliorates allergic airway inflammation in vivo. Hence, our study provides mechanistic insights into the inhibitory effect of IL-4 on 
A

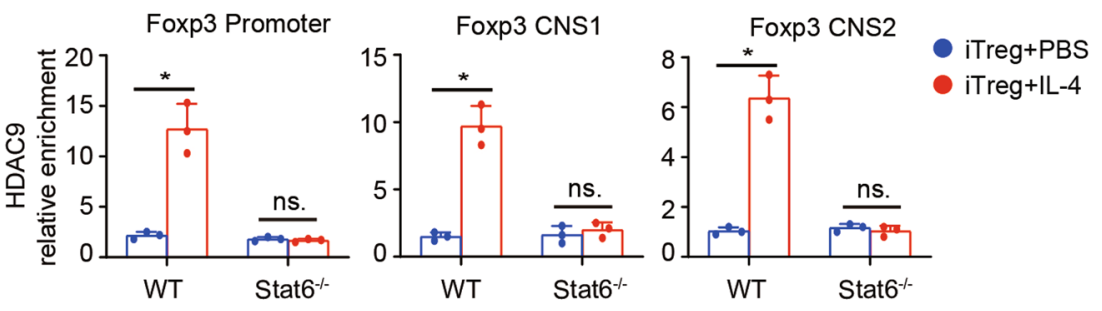

B

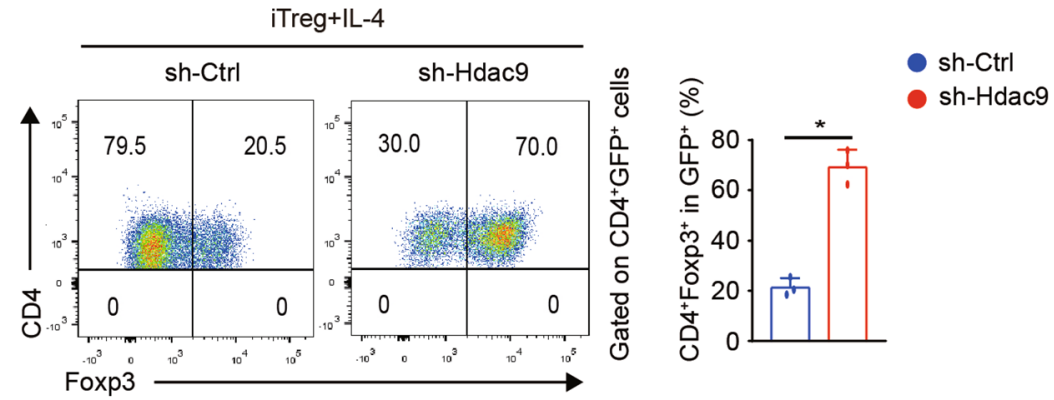

C
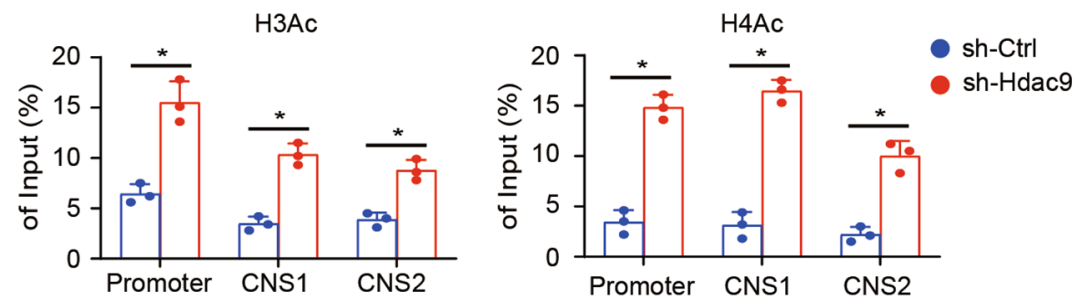

Fig. 5 Hdac9 knockdown abrogates the Foxp3 suppression of IL-4. A ChIP-qPCR assays for HDAC9 enrichment in the Foxp3 promoter, CNS1 and CNS2 regions. B CD4 ${ }^{+} T$ cells were transfected with a retrovirus carrying Hdac9-specific or Ctrl shRNA and cultured under iTreg-polarizing conditions with IL-4. On day 3, FCM assay was used to measure the percentages of Foxp3 ${ }^{+}$cells among retrovirus-transfected CD4 ${ }^{+} \mathrm{T}_{\text {cells }}\left(\mathrm{GFP}{ }^{+}\right)$. C ChIP-qPCR assays for H3Ac and H4Ac modifications in the Foxp3 promoter, CNS1 and CNS2 regions were performed with retrovirus-transfected CD4 ${ }^{+}$T cells on day 2. Data are the mean \pm SD and representative of three independent experiments. ${ }^{*} p<0.05$.

Treg induction and a potential therapeutic strategy for the field of allergic airway disease.

The mechanisms regulating Foxp3 gene transcription are relatively complex and involve a series of transcription factors ${ }^{27}$. As a classic downstream molecule of IL-4, STAT6 is involved in the transcriptional inhibition of Foxp3 in some way. Although it has been reported that the Foxp3 locus contains a STAT6 binding site ${ }^{28}$, the mechanism by which STAT6 inhibits Foxp3 gene transcription has not yet been clarified. On the other hand, there is an emerging area of great significance concerning mechanisms of epigenetic regulation. We provide compelling evidence that chromatin modifications play an important role in the suppression process, and STAT6 is indispensable for this process. In addition, there have been some controversies about the necessity and role of GATA3 in this process ${ }^{10,28,29}$. Our results indicate that GATA3 is dispensable for the inhibition of Foxp3 transcription, although the GATA3 level did increase with the addition of IL-4. These contradictions reflect the complex relationship among STAT6, GATA3, and Foxp3.

There are four classes of HDACs: class I includes HDAC1, HDAC2, HDAC3, and HDAC8, class II includes subclasses IIa (HDAC4, HDAC5, HDAC7, and HDAC9) and IIb (HDAC6 and HDAC10), class III includes the HDACs SIRT1-7, and HDAC11 is the class IV HDAC ${ }^{30}$. In the HDACi screening assay, we found that the effects of class I HDAC-specific inhibitors were not as strong as those of pan-HDACi and class II HDAC-specific inhibitors, indicating that class II HDAC plays a main role in the suppression induced by IL-4. Then, we targeted HDAC9 through a series of experiments. Indeed, HDAC9 has the highest expression among class II HDACs, and $\mathrm{Hdac}^{-/-}$mice have increased numbers of $\mathrm{Tregs}^{23}$. Of note, class II HDACs normally do not show strong deacetylase activity and instead act mainly as scaffolding ${ }^{19}$. 
A

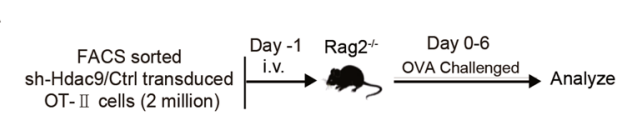

C

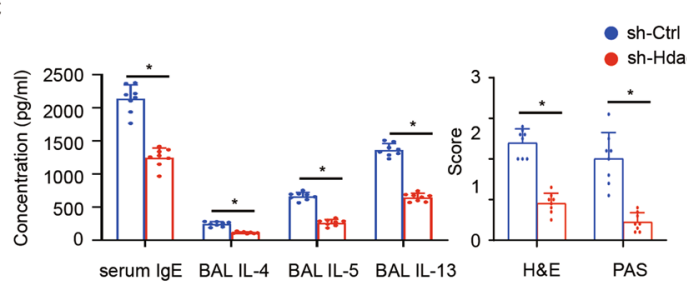

B

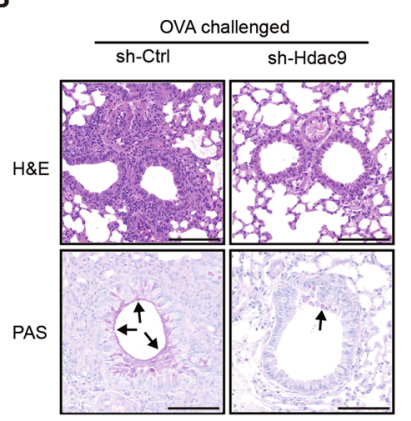

D

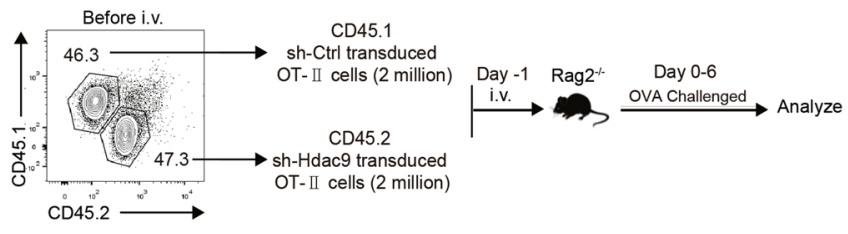

E

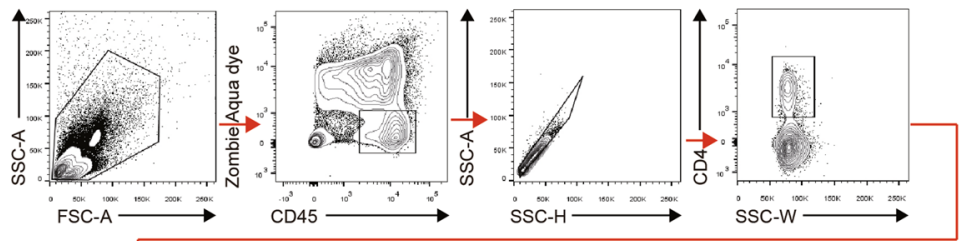

$\mathbf{F}$

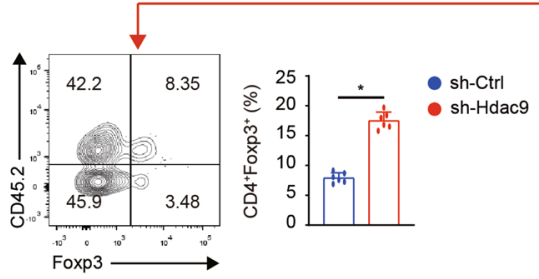

Fig. 6 Hdac9 knockdown alleviates allergic airway inflammation and increases Treg proportion in an OT-Il cell adoptive transfer model. A Schematic representation of the Hdac9-knockdown OT-II cell transfer allergy model. B Representative lung histology sections displaying inflammatory cell infiltration and PAS ${ }^{+}$mucin-secreting cell hyperplasia. The arrow indicates purple-red mucin-producing cells. Scale bars, $100 \mu \mathrm{m}$. C Total IgE levels in the blood serum and IL-4, IL-5, and IL-13 levels in the BAL fluid, as determined by ELISA. Graph displaying the histological scores determined by H\&E and PAS staining. Five areas were randomly chosen in each slide. D Schematic representation of the CD $45.1 / 2^{+}$sh-Ctrl/Hdac9 OT-II cell transfer allergy model. E Gating strategy of CD4 ${ }^{+}$Foxp3 $^{+}$Tregs infiltrated in lungs. $\mathbf{F}$ The percentages of Foxp3 ${ }^{+}$cells among CD45.1 ${ }^{+}$or CD45.2 $2^{+} \mathrm{CD}^{+} \mathrm{T}$ cells were displayed by contour plot and histogram. The Each group included six to eight mice. Data are the mean \pm SD and representative of three independent experiments. ${ }^{*} p<0.05$.

Therefore, further study is needed to determine whether other proteins, such as other HDACs, are involved in this IL-4-induced suppression. Moreover, the detailed regulatory mechanism linking STAT6 and HDAC9 need to be clarified.

In addition to their well-known effects on malignant cells, including proapoptotic activity or cell cycle arrest inductions, some beneficial effects on autoimmune diseases, including colitis and arthritis, have been discovered for $\mathrm{HDACi}^{23,31}$. Mechanistically, HDACi can increase the number of Tregs and enhance their function, possibly through hyperacetylation of histones in Tregs ${ }^{32}$, which is in accordance with our research. Moreover, the panHDACi TSA has been reported to prolong the survival time of MHC-mismatched mouse heart allografts ${ }^{33}$. This drove us to study the effect of $\mathrm{NaB}$ on transplant immunoreaction. In acute transplant immunoreaction, $\mathrm{NaB}$ showed little effect on both skin and heart allografts survival (sFig 2A, B). Then we focused on chronic transplant immunoreaction, which is transplant-associated obliterative bronchiolitis. The results showed that $\mathrm{NaB}$ ameliorated obliterative bronchiolitis in a mouse tracheal transplantation model (sFig 2C), indicating the therapeutic potential of $\mathrm{NaB}$ in the field of transplantation. Nevertheless, the application of this pan-HDACi has been limited due to extensive side effects ${ }^{34}$. Hence, the development of a specific HDAC9 inhibitor might contribute to addressing this issue. 
Our research provides new explanations for the mechanisms underlying the inhibition of Treg induced by IL-4, indicating the important role of epigenetic changes in this process. The pan-HDACi $\mathrm{NaB}$ might be a potential therapeutic for the treatment of asthma patients and transplant recipients.

\section{STAR methods}

Animals

Stat6 $^{-1-}$, Rag2 ${ }^{-1-}$, and OT-II mice were acquired from The Jackson Laboratory (Bar Harbor, ME), and wild-type (WT) C57BL/6 mice were purchased from Shanghai Model Organisms (Shanghai, China). All animals were maintained in a specific pathogen-free barrier facility at Tongji Medical College, Huazhong University of Science and Technology. All animal use and care were approved by the Institutional Animal Care and Use Committee of Tongji Medical College.

\section{Acute allergic lung inflammation model}

Female C57BL/6 mice (8 to 10-weeks-old) were injected intraperitoneally (i.p.) with $100 \mu \mathrm{l}$ sensitizing solution on days 0,7 , and 14 . The sensitizing solution was composed of $20 \mathrm{mg}$ ovalbumin (OVA) (A5503; Sigma) and $4 \mathrm{~g}$ aluminum hydroxide (A510023; Sangon Biotech) dissolved in $100 \mathrm{ml}$ PBS. From days 21 to 27, the sensitized mice were i.p. injected with $250 \mu \mathrm{l}$ PBS or $1 \mathrm{M}$ sodium butyrate dissolved in PBS (A510838; Sangon Biotech) ) $^{35,36}$ and exposed to aerosolized OVA (1\% in PBS) for $40 \mathrm{~min}$ every day. In an adoptive transfer model, FACS-sorted shHdac9- or shCtrl-transduced OT-II CD45.1/2 T cells were intravenously (i.v.) transferred into $\mathrm{Rag} 2^{-1-}$ mice ( $2 \mathrm{million} / \mathrm{mouse}$ ), and then the transferred mice were treated with aerosolized OVA (1\% in PBS) for $40 \mathrm{~min} \times$ 7 day $^{37}$. The next day, all the parameters for airway allergy were measured. Specifically, bronchoalveolar lavage (BAL) was performed by cannulating the trachea with a polyethylene tube and $1 \mathrm{ml}$ sterile PBS, and the BAL fluid was processed by centrifugation at $500 \mathrm{~g}$ to isolate cells from the supernatant. The supernatant was used to evaluate IL-4, IL-5, and IL-13 levels with ELISA kits (IL-4 and IL-5, Dakewe; IL-13, Abclonal). The cells in the BAL fluid were stained with trypan blue and a Hema 3 staining kit (Fisher Scientific) to assess total and differential cell counts, respectively. The total IgE level in the blood was determined with an ELISA kit (Dakewe). Inflammatory cells in lung tissues were collected with 40\% Percoll (P8370; Solarbio) after the tissues were ground. Lung tissues in the same anatomical location were made into sections and subjected to hematoxylin and eosin (H\&E) and periodic acid-Schiff (PAS) staining. A semiquantitative scoring system was adopted for quantification of tissue histopathology ${ }^{38}$.

\section{In vitro $T$ cell stimulation}

FACS-sorted naive $\mathrm{CD}^{+} \quad \mathrm{T}$ cells $\left(\mathrm{CD} 62 \mathrm{~L}^{\text {high }} \mathrm{C}\right.$ $\mathrm{D} 44^{\text {low }} \mathrm{CD} 25^{-}, 1 \times 10^{5}$ cells/well) were activated with plate-bound anti-CD3e monoclonal antibodies (mAbs; $5 \mu \mathrm{g} / \mathrm{ml}$, clone 2C11, BioLegend) and soluble anti-CD28 $\mathrm{mAbs}(1 \mu \mathrm{g} / \mathrm{ml}$, clone 37.51 , BioLegend $)$ in 96-well tissue culture plates (Biofil). For induction of Tregs in vitro, TGF- $\beta 1(5 \mathrm{ng} / \mathrm{ml})$ and IL-2 $(10 \mathrm{ng} / \mathrm{ml})$ were added to the medium; in some experiments, IL-4 $(15 \mathrm{ng} / \mathrm{ml})$ was added. All recombinant cytokines were obtained from PeproTech. The following inhibitors were added at respective concentrations: pan-HDAC inhibitors trichostatin A (TSA) (HY-15144), NaB (HY-B0350A), and SAHA (HY-10221); class I/IV HDAC inhibitor AES-135 (HY-114483); class I HDAC inhibitor MS-275 (HY-12163); HDAC3 inhibitor RGFP966 (HY-13909); HDAC6 inhibitor CG347B (HY135890); HDAC8 inhibitor HDAC8-IN-1 (HY-111342); SIRT1 inhibitor EX-527 (HY-15452); SIRT3 inhibitor 3-TYP (HY-108331); SIRT inhibitor SIRT-IN-3 (HY$133998)^{17,39,40}$, all the inhibitors were purchased from MedChemExpress; class IIa HDAC inhibitor TMP269 (S7324, Selleck). After polarization for 1-3 days, the $\mathrm{T}$ cells were collected for different analyses.

\section{Assay for transposase-accessible chromatin with high- throughput sequencing (ATAC-seq)}

Naive $\mathrm{CD} 4^{+} \mathrm{T}$ cells $\left(\mathrm{CD} 62 \mathrm{~L}^{\text {high }} \mathrm{CD} 44^{\text {low }} \mathrm{CD} 25^{-}\right)$were sorted from the spleen of 6 to 8-week-old male WT C57BL/6 on a FACSAria II (BD) and stimulated with platebound anti-CD3 $(5 \mu \mathrm{g} / \mathrm{ml}, 145-2 \mathrm{C} 11$; BioLegend) plus soluble anti-CD28 antibodies $(1 \mu \mathrm{g} / \mathrm{ml}, 37.51$; BioLegend $)$ in the presence of TGF- $\beta 1$ ( $5 \mathrm{ng} / \mathrm{mL}$; PeproTech) and mouse IL-2 $(10 \mathrm{ng} / \mathrm{mL}$; PeproTech) with or without mouse IL-4 (15 ng/mL; PeproTech) for $48 \mathrm{~h}$. Sample processing and library preparation were performed following instructions described previously ${ }^{41}$. Then, the samples were sequenced on the BGISEQ-500 platform (BGI-Shenzhen, China). Clean paired-end reads were mapped to the $\mathrm{mm} 10$ reference genome by means of Bowtie2 (v2.2.5) ${ }^{42}$; only mapped pairs reads were kept for further analysis. We used MACS2 (v2.1.2) to call peaks (open chromatin regions) using a parameter described previously ${ }^{41}$. Finally, the BedGraph file was converted into a normalized BigWig file for visualization in Integrative Genomics Viewer (IGV).

\section{Intracellular staining}

FCM was performed as previously reported ${ }^{43}$. For cytokine staining, $\mathrm{T}$ cells were briefly restimulated with phorbol 12-myristate 13-acetate (50 ng/ml; Sigma-Aldrich) and ionomycin (550 ng/ml; Sigma-Aldrich) with the addition of GolgiStop (BD Pharmingen) for $4 \mathrm{~h}$. After fixation and permeabilization with a Foxp3 staining buffer set (eBioscience) according to the manufacturer's instructions, the cells were stained with anti-IL-9 (RM9A4), anti-Foxp3 
(FJK-16s), and anti-GATA3 (16E10A23) antibodies. Other FCM antibodies used in this study included anti-CD4 (GK1.5), anti-CD45 (30-F11), anti-CD11c (N418), anti-F4/ 80 (T45-2342), and anti-Siglec F (S17007L). All samples were acquired with a BD LSR Fortessa X-20 flow cytometer. The results were analyzed using FlowJo v10 software (Tree Star, Inc.).

\section{Quantitative RT-PCR}

Total RNA was extracted from samples using an RNAprep Pure Cell/Bacteria kit (S7717; Tiangen) and reverse transcribed into cDNA with ABScript II RT Master Mix for qPCR with gDNA Remover (RK20403; ABclonal). Using specific primers for target genes (Supplementary Table 1), quantitative real-time PCR was performed with UltraSYBR Mixture (CW0957; CoWin Biosciences) and a Bio-Rad CFX96 real-time PCR system. The relative expression of target genes was calculated with the 2- $\Delta \Delta \mathrm{Ct}$ method after normalization to the expression of the Gapdh gene.

\section{Immunoblot analysis}

After polarization for 3 days, T cells were lysed in RIPA lysis buffer for $10 \mathrm{~min}$ on ice and then disposed with ultrasonic wave. The lysate was centrifuged at $12,000 \mathrm{~g}$ for $5 \mathrm{~min}$ at $4{ }^{\circ} \mathrm{C}$, then resuspended in sample buffer and boiled at $95^{\circ} \mathrm{C}$ for $10 \mathrm{~min}$ to prepare it for following SDSPAGE fractionation and transfer. The following specific antibodies were used for immunoblot analysis: antiHDAC9 (ab59718; 1:1,000; Abcam) and anti- $\beta$-Actin (BM5180; 1:1,000; BOSTER).

\section{Chromatin immunoprecipitation assay}

After polarization for $48 \mathrm{~h}$ under different conditions, $\mathrm{CD}^{+} \mathrm{T}$ cells were processed with an EZ ChIP kit (17-371; EMD Millipore). Anti-H3Ac (39139; $7 \mu \mathrm{l})$, anti-H4Ac (39243; $6 \mu \mathrm{l})$, anti-H3K9Me3 (61013; $7 \mu \mathrm{l})$, anti-H3K27Me2 $(39245 ; 5 \mu \mathrm{l}$; all from Active Motif), anti-H3K27Me3 (ab6002; $5 \mu \mathrm{l})$, anti-H3K27Ac (ab4729; $3 \mu \mathrm{l})$, anti-H3K9Ac (ab4441; $4 \mu \mathrm{l}$; all from Abcam), anti-H3K9Me2 (D5567; $5 \mu \mathrm{l}$; Sigma-Aldrich), anti-HDAC9 (ab59718; $5 \mu \mathrm{g} ;$ Abcam) and purified rabbit IgG (A7016; $5 \mu$ g; Beyotime) antibodies were used for chromatin immunoprecipitation. Then, the precipitated DNA was evaluated by quantitative RT-PCR as described earlier. The primer sequences used for quantitative RT-PCR are listed in Supplementary Table 1. Relative binding was calculated based on normalization to the input DNA.

\section{shRNA-mediated gene knockdown in $T$ cells}

With an online tool (https://rnaidesigner.thermofisher. com), we designed and synthesized shRNA sequences to target Hdac9 at Tsingke Biological Technology. Retroviral particles were prepared as previously described ${ }^{40}$.
To select the most efficient shRNA for further experiments, NIH-3T3 cells and WB were used to evaluate knockdown efficiency. Naive $\mathrm{T}$ cells were activated for $24 \mathrm{~h}$ with plate-bound anti-CD3e mAbs $(5 \mu \mathrm{g} / \mathrm{ml})$ and soluble anti-CD28 mAbs $(1 \mu \mathrm{g} / \mathrm{ml})$ and then centrifuged for $2 \mathrm{~h}$ at $780 \mathrm{~g}$ and $32{ }^{\circ} \mathrm{C}$ with the retroviral particles and $8 \mathrm{mg} / \mathrm{ml}$ polybrene, followed by incubation for $6 \mathrm{~h}$ at $32^{\circ} \mathrm{C}$. Subsequently, the cells were cultured under various polarization conditions in complete RPMI 1640 medium at $37^{\circ} \mathrm{C}$. The shRNA-transduced $\mathrm{T}$ cells $\left(\mathrm{GFP}^{+}\right)$were sorted by FACS, and HDAC9 expression was analyzed by WB. The sequence of the HDAC9-specific shRNA used was $5^{\prime}$ GCTCAAGATAGCAAGGATGAT 3'.

\section{Statistics}

Data were analyzed with GraphPad Prism 8 represented as mean \pm SD. Statistical analyses were completed with unpaired Student's $t$ test between the two groups and oneway analysis of variance (ANOVA) with least significant difference test among three or more groups. Significance was considered when $p<0.05 . p$ values are represented in this article as follows: ns. $p \geq 0.05$; ${ }^{*} p<0.05$.

\section{Author contributions}

J.X. and J.W. conceived and designed the experiments; J.C. and J.Y. drafted the manuscript; J.C., J.Y., and H.X. performed the in vitro experiments; X.Z., Y.D., and Y.Z. established the animal models; H.X., Z.C., and Y.L. analyzed the data. All authors read the manuscript and approved the final version of the manuscript.

\section{Funding}

This work was supported by the National Natural Science Foundation of China (82071803, 81730015), Natural science fund of Hubei Province (2019AAA032), and the Fundamental Research Funds for the Central Universities', (HUST No. 2021GCRC037).

\section{Data availability}

The accession numbers for the ATAC-seq data reported in this paper are GEO: GSE152810.

Conflict of interest

The authors declare no competing interests.

Ethics statement

All animal use and care were approved by the Institutional Animal Care and Use Committee of Tongji Medical College.

\section{Publisher's note \\ Springer Nature remains neutral with regard to jurisdictional claims in published maps and institutional affiliations.}

Supplementary information The online version contains supplementary material available at https://doi.org/10.1038/s41419-021-03769-7.

Received: 22 December 2020 Revised: 27 April 2021 Accepted: 28 April 2021

Published online: 18 May 2021

References

1. Sakaguchi, S., Yamaguchi, T., Nomura, T. \& Ono, M. Regulatory T cells and immune tolerance. Cell 133, 775-787 (2008).

2. Curotto de Lafaille, M. A. \& Lafaille, J. J. Natural and adaptive foxp3+ regulatory T cells: more of the same or a division of labor? Immunity 30, 626-635 (2009). 
3. Hori, S., Nomura, T. \& Sakaguchi, S. Control of regulatory T cell development by the transcription factor Foxp3. Science 299, 1057-1061 (2003).

4. Brunkow, M. E. et al. Disruption of a new forkhead/winged-helix protein scurfin, results in the fatal lymphoproliferative disorder of the scurfy mouse. Nat. Genet 27, 68-73 (2001).

5. Fontenot, J. D. \& Rudensky, A. Y. A well adapted regulatory contrivance: regulatory $\mathrm{T}$ cell development and the forkhead family transcription factor Foxp3. Nat. Immunol. 6, 331-337 (2005).

6. Chen, W. et al. Conversion of peripheral CD4+CD25- naive T cells to CD4 $+\mathrm{CD} 25+$ regulatory $\mathrm{T}$ cells by TGF-beta induction of transcription factor Foxp3. J. Exp. Med 198, 1875-1886 (2003).

7. Burchill, M. A., Yang, J., Vogtenhuber, C., Blazar, B. R. \& Farrar, M. A. IL-2 receptor beta-dependent STAT5 activation is required for the development of Foxp3+ regulatory T cells. J. Immunol. 178, 280-290 (2007).

8. Zhou, X. et al. Instability of the transcription factor Foxp3 leads to the generation of pathogenic memory T cells in vivo. Nat. Immunol. 10, 1000-1007 (2009).

9. Bettelli, E. et al. Reciprocal developmental pathways for the generation of pathogenic effector TH17 and regulatory T cells. Nature 441, 235-238 (2006)

10. Dardalhon, V. et al. IL-4 inhibits TGF-beta-induced Foxp3+ T cells and, together with TGF-beta, generates IL-9+ IL-10+ Foxp3(-) effector T cells. Nat Immunol. 9, 1347-1355 (2008).

11. Noval Rivas, M. et al. Regulatory T cell reprogramming toward a Th2-cell-like lineage impairs oral tolerance and promotes food allergy. Immunity $\mathbf{4 2}$ 512-523 (2015).

12. Lambrecht, B. N., Hammad, H. \& Fahy, J. V. The cytokines of asthma. Immunity 50, 975-991 (2019).

13. Koh, B. et al. Bcl6 and Blimp1 reciprocally regulate ST2(+) Treg-cell development in the context of allergic airway inflammation. J Allergy Clin Immunol. 146, 1121-1136 (2020).

14. Kanno, Y., Vahedi, G., Hirahara, K., Singleton, K. \& O'Shea, J. J. Transcriptional and epigenetic control of $\mathrm{T}$ helper cell specification: molecular mechanisms underlying commitment and plasticity. Annu Rev. Immunol. 30, 707-731 (2012).

15. Lam, A. L., Pazin, D. E. \& Sullivan, B. A. Control of gene expression and assembly of chromosomal subdomains by chromatin regulators with antagonistic functions. Chromosoma 114, 242-251 (2005).

16. Tian, L. et al. Reversible histone acetylation and deacetylation mediate genome-wide, promoter-dependent and locus-specific changes in gene expression during plant development. Genetics 169, 337-345 (2005).

17. Bantscheff, M. et al. Chemoproteomics profiling of HDAC inhibitors reveals selective targeting of HDAC complexes. Nat. Biotechnol. 29, 255-265 (2011).

18. Yoo, C. B. \& Jones, P. A. Epigenetic therapy of cancer: past, present and future Nat. Rev. Drug Disco. 5, 37-50 (2006).

19. Akimova, T., Beier, U. H., Liu, Y., Wang, L. \& Hancock, W. W. Histone/protein deacetylases and T-cell immune responses. Blood 119, 2443-2451 (2012).

20. Chen, S. et al. Epigenetically modifying the Foxp3 locus for generation of stable antigen-specific Tregs as cellular therapeutics. Am. J. of transpl. 20, 2366-2379 (2020)

21. von Knethen A., Heinicke U., Weigert A., Zacharowski K. \& Brune B. Histone deacetylation inhibitors as modulators of regulatory T cells. Int. J. Mol. Sci. 21, $2356(2020)$

22. Tao, R. et al. Deacetylase inhibition promotes the generation and function of regulatory T cells. Nat. Med 13, 1299-1307 (2007).
23. de Zoeten, E. F., Wang, L., Sai, H., Dillmann, W. H. \& Hancock, W. W. Inhibition of HDAC9 increases $T$ regulatory cell function and prevents colitis in mice. Gastroenterology 138, 583-594 (2010).

24. Ghonim, M. A. et al. Sulfated non-anticoagulant heparin blocks Th2-induced asthma by modulating the IL-4/signal transducer and activator of transcription 6/Janus kinase 1 pathway. J. Transl. Med 16, 243 (2018).

25. Allinne, J. et al. IL-33 blockade affects mediators of persistence and exacerbation in a model of chronic airway inflammation. J. Allergy Clin. Immunol. 144, 1624-1637.e10 (2019).

26. Zhu, J., Yamane, H. \& Paul, W. E. Differentiation of effector CD4 T cell populations (*). Annu. Rev. Immunol. 28, 445-489 (2010).

27. Tone, M. \& Greene, M. I. Cooperative regulatory events and Foxp3 expression. Nat. Immunol. 12, 14-16 (2011).

28. Takaki, H. et al. STAT6 Inhibits TGF-beta1-mediated Foxp3 induction through direct binding to the Foxp3 promoter, which is reverted by retinoic acid receptor. J. Biol. Chem. 283, 14955-14962 (2008).

29. Mantel, P. Y. et al. GATA3-driven Th2 responses inhibit TGF-beta1-induced FOXP3 expression and the formation of regulatory T cells. PLoS Biol. 5, e329 (2007).

30. Haberland, M., Montgomery, R. L. \& Olson, E. N. The many roles of histone deacetylases in development and physiology: implications for disease and therapy. Nat. Rev. Genet 10, 32-42 (2009).

31. Saouaf, S. J. et al. Deacetylase inhibition increases regulatory $T$ cell function and decreases incidence and severity of collagen-induced arthritis. Exp. Mol. Pathol. 87, 99-104 (2009).

32. Wang, L, de Zoeten, E. F. Greene, M. I. \& Hancock, W. W. Immunomodulatory effects of deacetylase inhibitors: therapeutic targeting of FOXP3 + regulatory T cells. Nat. Rev. Drug Disco. 8, 969-981 (2009).

33. Wang, L., Tao, R. \& Hancock, W. W. Using histone deacetylase inhibitors to enhance Foxp3 $(+)$ regulatory T-cell function and induce allograft tolerance. Immunol. Cell Biol. 87, 195-202 (2009).

34. Peng, X., Liao, G., Sun, P., Yu, Z. \& Chen, J. An overview of HDAC inhibitors and their synthetic routes. Curr. Top. Med Chem. 19, 1005-1040 (2019).

35. Tyagi, A. M. et al. The microbial metabolite butyrate stimulates bone formation via T regulatory cell-mediated regulation of WNT10B expression. Immunity 49, 1116-1131.e7 (2018)

36. Vieira, R. S. et al. Butyrate attenuates lung inflammation by negatively modulating Th9 cells. Front Immunol. 10, 67 (2019).

37. Xiao, X. et al. Guidance of super-enhancers in regulation of IL-9 induction and airway inflammation. J. Exp. Med 215, 559-574 (2018).

38. Ventura, S. et al. A20-binding inhibitor of NF-kappaB (ABIN) 2 negatively regulates allergic airway inflammation. J. Exp. Med 215, 2737-2747 (2018).

39. Xiao, X. et al. GITR subverts Foxp3(+) Tregs to boost Th9 immunity through regulation of histone acetylation. Nat. Commun. 6, 8266 (2015)

40. Zhang, $X$. et al. OX40 costimulation inhibits Foxp3 expression and treg induction via BATF3-dependent and independent mechanisms. Cell Rep. 24 607-618 (2018)

41. Schwartz, D. M. et al. Retinoic acid receptor alpha represses a Th9 transcriptional and epigenomic program to reduce allergic pathology. Immunity $\mathbf{5 0}$ 106-120.e10 (2019)

42. Langmead, B. \& Salzberg, S. L. Fast gapped-read alignment with Bowtie 2. Nat Methods 9, 357-359 (2012)

43. Cui, J. et al. Autophagy-lysosome inhibitor chloroquine prevents CTLA-4 degradation of $\mathrm{T}$ cells and attenuates acute rejection in murine skin and heart transplantation. Theranostics 10,8051-8060 (2020). 